\title{
Magnetic Anisotropy in Silicon Iron Alloys
}

\author{
Veronica Paltanea, Gheorghe Paltanea*, Horia Gavrila
}

Department of Electrical En gineering, POLITEHNICA University from Bucharest, Bucharest, Romania

\begin{abstract}
In this article it is presented a study of the magnetic anisotropy of non-oriented and grain oriented Fe-Si strips with the surface area of $280 \times 30 \mathrm{~mm}^{2}$. The measurements were performed with a unidirectional single strip tester on Fe-Si strips cut at $0^{\circ}, 15^{\circ}, 30^{\circ}, 45^{\circ}, 60^{\circ}, 75^{\circ}, 90^{\circ}$ with the rolling direction. For the representation of the magnetic field strength at constant magnetic flux density it was used a software program that interpolated the experimental results. It was determined the hard and the easy axis of the samples and the influence of the frequency on the anisotropy of the materials.The loss behaviour of these alloys was studied as a function of frequency in the range of $5 \mathrm{~Hz}$ to $200 \mathrm{~Hz}$ at a given magnetic polarization $\left(J_{p}\right)$ of $1 \mathrm{~T}$ and using the concept of loss separation for the data analysis it was given a corre lation between the different types of losses and the anisotropy axis.
\end{abstract}

Keywords Magnetic Anisotropy, Silicon Iron Strips, Magnetic Domains, Rolling Direction

\section{Introduction}

Grain oriented (GO) and non-oriented (NO) laminations owe their continuing importance as a unique subject of basic research studies and industrial applications to their excellent crystallographic properties[1]. While in many cases physical modelling has been limited to the properties related to the rolling direction, the use of computational methods in magnetic cores call for the knowledge of magnetization curve and hysteresis loops on different axis fro $m$ the rolling direction $[2,3]$.

For evaluating and controlling the quality of magnetic materials, magnetic properties, such as $B(H)$ curves and core losses, are measured under one-dimensional (1D) alternating and/or two-dimensional (2D) rotating magnetic fluxes, according to the application requirement.The standard magnetic measurements using either the Epstein frame or the single strip tester are limited to the rolling direction measurements for the GO Fe-Si materials. For the NO ones, standard measurements give an average of the magnetic behaviour in the rolling and the transverse directions. The hysteresis in the rolling direction $B_{L}\left(H_{L}\right)$ in the case of Fe-Si GO strips and an average one in the case of Fe-Si NO stripswere obtained.The total error of any magnetic measurement depends on the exciting field error, the error of the magnetic to electric variables conversion and the error of integral electric values measurement [4-6].

Thin anisotropic electrical strips (Fe-Si GO) are manufactured by cold rolling of low carbon steel containing

\footnotetext{
* Corresponding author:

paltanea03@yahoo.com (Gheorghe Paltanea)

Published online at http://journal. sapub.org/eee

Copyright $\odot 2012$ Scientific \& Academic Publishing. All Rights Reserved
}

about $3.5 \mathrm{wt} \% \mathrm{Si}$. The crystallites of Fe-Si GO laminations have their[001] easy axis closed to the rolling direction and their (110) plane nearly parallel to the laminations surface. This is so-called Goss texture which can lead to coercivefieldsas low as $4-10 \mathrm{~A} / \mathrm{m}$ and a maximum relativepermeabilityaround $5 \times 10^{4}$. GO laminations are subdivided in two classes: conventional grain oriented (CGO) and high permeability (HGO)[7, 8]. In this article it was used CGO materials which have the following preparation steps: first the composition is melted, vacuum degassed and casted in slabs; theslabs are reheated at $1300-1350{ }^{\circ} \mathrm{C}$ and hot rolled to the thickness of $2 \mathrm{~mm}$, annealed at $900-1100{ }^{\circ} \mathrm{C}$ and rapid cooled; after that it is done a two-stage cold rolling to the final gauge; in the end it is done a decarburizing anneal in wet $\mathrm{H}_{2}$ atmosphere at 800-850 ${ }^{\circ} \mathrm{C}$, followed by cooling and an 48 hours box annealing at $1200{ }^{\circ} \mathrm{C}[8]$. They are produced in a variety of sizes, ranging between 0.05 and $0.20 \mathrm{~mm}$ thickness and between 25 and $120 \mathrm{~mm}$ in width. Anisotropic strips exhib it relatively low magnetic losses (being a function of thickness, magnetic induction, resistivity and frequency) and large magnetic induction in their easiest direction of magnetization. These strips are manufactured in small amounts for a typical use in wound or stacked magnetic cores working at high frequencies or at impulse conditions [7].

The non-oriented $\mathrm{Fe}-\mathrm{Si}$ alloys (Fe-Si NO) have an approximately isotropic grain texture, but they present a slightly anisotropy with the easy axis parallel with rolling directions and hard axis perpendicular of the rolling directions [6]. They are available in a variety of grades, the higher ones being as sociated with higher Si content. The Si concentration can vary between 1 and $3.7 \mathrm{wt} \%$ which increased the alloy resistivity without impairing the 
mechanical properties. Lower grade laminations up to 2 $\mathrm{wt} \% \mathrm{Si}$ are produced in the semi-processed state with thickness between 0.5 and $0.65 \mathrm{~mm}$. The preparation stages of the Fe-Si NO strips include a cold rolled to intermediate gauge,annealed at $750-900^{\circ} \mathrm{C}$ to reduce to the final gauge of $0.35-0.65 \mathrm{~mm}$. The strips are then subjected to a recrystallization and decarburization anneal at $830-900{ }^{\circ} \mathrm{C}$ and a final grain-g rowth anneal at $850-1100^{\circ} \mathrm{C}$ [8].

Such thin electrical strips display beneficial magnetic properties both at high frequencies and at impulse work conditions. Some applications imply the characterization of $\mathrm{Fe}-\mathrm{Si}$ grain-oriented and non-oriented laminations along a direction different from rolling direction. This serves to emulate, for instance, the working conditions in certain parts of electrical machine cores[5]. In materials such as Fe-Si strips magnetization is determined by the motion of $180^{\circ}$ and $90^{\circ}$ walls until the net force on all walls is zero [8].

In order to measure the magnetic characteristics of the electrical steel strips with a double C-yoke single strip tester, the samples were properly prepared and placed in a magnetic field, created by a coil system. The measuring system consists of a primary coil that generates a magnetic field, which causes the magnetic flux in the tested strip. The magnetic field generator is a solenoid of rectangular cross section, dimensioned to ensure the generation of a magnetic field, intense enough to bring the sample into a desired state of magnetization, in which are measured the energy losses. An important requirement is that the solenoid must ensure a high uniformity of the magnetic field, applied along the sample in the measurement area.

The magnetic field strength is determined by the number of windings and the length of the coil. The number of turns and the length are determined by measuring the geometry of the apparatus. The magnetic field strength is calculated from the electrical current, picked from a shunt of known value, connected in series with the magnetizing coil.

The magnetic flu $x$ density is determined by measuring the voltage, induced in the secondary coil of the measuring system.

The measurement of the magnetic properties is made with a sinusoidal magnetic flux density in accordance with DIN 50462 standards. The secondary voltage must also be sinusoidal.

\section{The Influence of the Frequency on the Anisotropy of Silicon Iron Strips}

The influence of frequency on the anisotropy of Fe-Si GO and NO strips was studied. Measurements at three frequencies $f=5,50,100 \mathrm{~Hz}$ were made and a comparison between the polar diagrams at a constant value of magnetic flux density $B$ was represented.

The variation of magnetic field for Fe-Si GO strips at $f=5$, $50 \mathrm{~Hz}$ at $\operatorname{low}(B=0.5 \mathrm{~T})$, medium $(B=1.02 \mathrm{~T})$ and high $(B=$ $1.5 \mathrm{~T})$ magnetic flux densities as a function of the cutting angleis represented in Figures 1, 2 and 3.

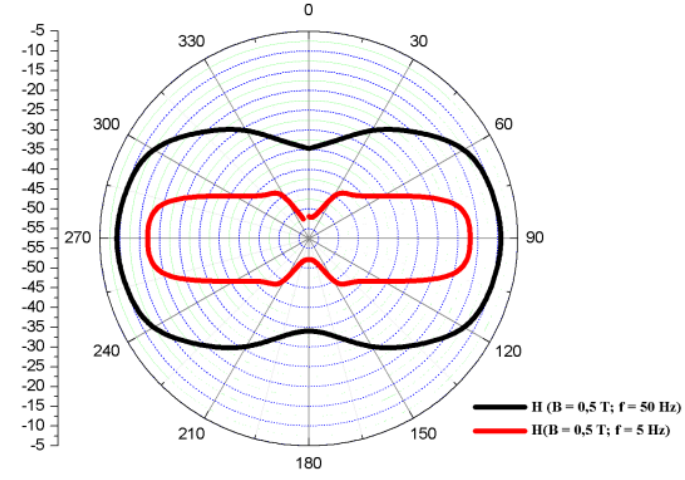

Figure 1. Comparison bet ween polar diagrams of the magnetic field in the case of Fe-Si GO strips at $B=0.5 \mathrm{~T}$ and frequencies $f=5,50 \mathrm{~Hz}$

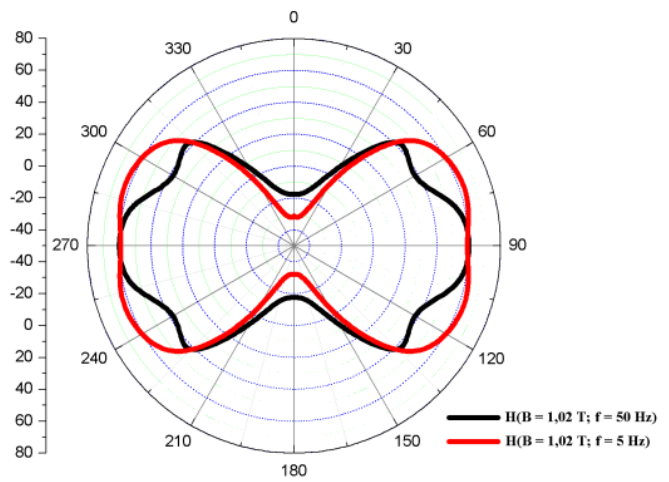

Figure 2. Comparison between polar diagrams of the magnetic field in the case of Fe-Si GO strips at $B=1.02 \mathrm{~T}$ and frequencies $f=5,50 \mathrm{~Hz}$

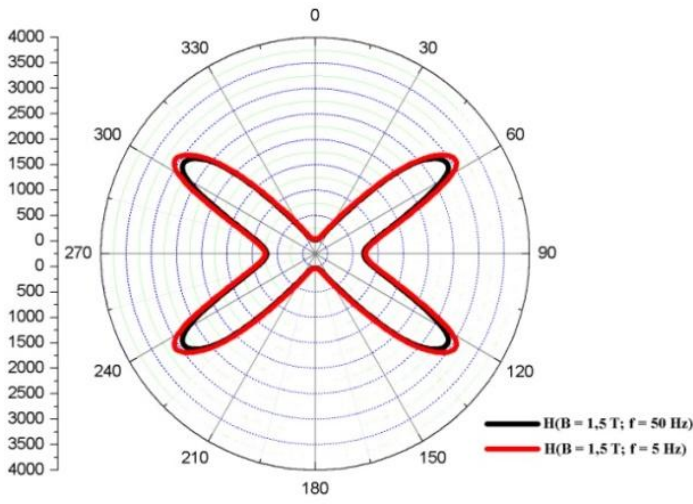

Figure 3. Comparison bet ween polar diagrams of the magnetic field in the case of Fe-Si GO strips at $B=1.5 \mathrm{~T}$ and frequencies $f=5,50 \mathrm{~Hz}$

It can be observed from Figures 1, 2,4 and 5 that hard magnetization axis is placed at $90^{\circ}$ and the easy axis is placed at $0^{\circ}$. The frequency does not modify the known easy and hard axis place $[5,6,9]$, which are influenced only by the value of magnetic flux density. In the case of the high magnetic flux density the hard axis is placed at $60^{\circ}$ and the easy axis at $0^{\circ}$ (Figures 3 and 6)[10]. At medium magnetic flux density, when the frequency is increasing, new domains were nucleated and the motion of already existing domain walls become s moother[11].

The variat ion of magnetic field for Fe-Si GO strips at $f=50$, $100 \mathrm{~Hz}$ at different magnetic flux densities is representedin Figures 4, 5 and 6. 


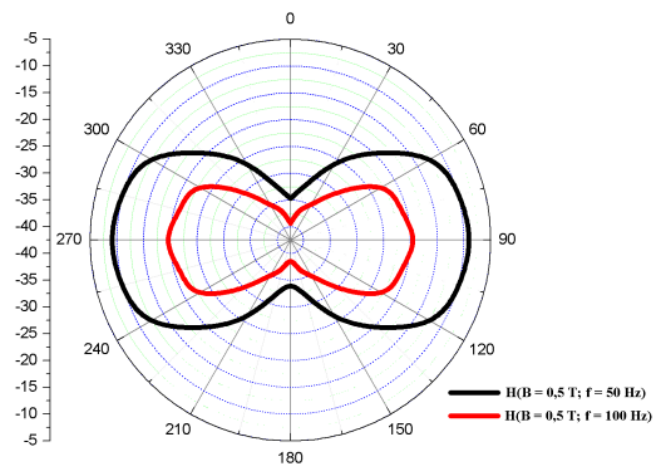

Figure 4. Comparison bet ween polar diagrams of the magnetic field in the case of Fe-Si GO strips at $B=0.5 \mathrm{~T}$ and frequencies $f=50,100 \mathrm{~Hz}$

At low values of magnetic flux density itresults the hard axis at $90^{\circ}$ and the easy axis at $0^{\circ}$, because in this case the orientation of the grain is not as important as in the interval of high induction.

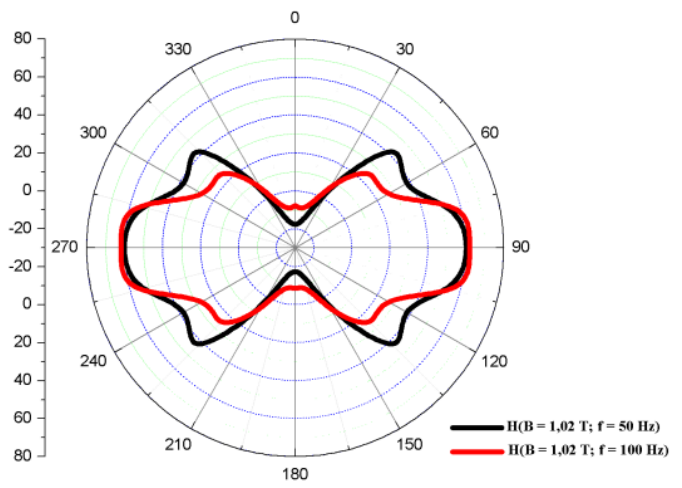

Figure 5. Comparison bet ween polar diagrams of the magnetic field in the case of Fe-Si GO strips at $B=1.02 \mathrm{~T}$ and frequencies $f=50,100 \mathrm{~Hz}$

In the case of polycrystalline materials of Goss texture, the orientation of an is otropy (hard and easy) a xes are at $0^{\circ}$, about $55^{\circ}-60^{\circ}$ (according to thetechnology of the strip production), and $90^{\circ}$.

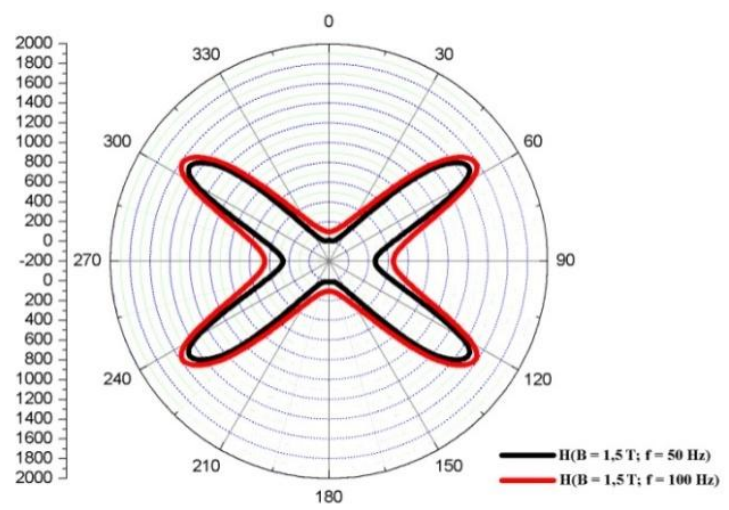

Figure 6. Comparison bet ween polar diagrams of the magnetic field in the case of Fe-Si GO strips at $B=1.5 \mathrm{~T}$ and frequencies $f=50,100 \mathrm{~Hz}$

As the texture (the preferred orientation of the crystallographic axes) of the polycrystalline material is done as a result of their plastic working, an optimum pattern of these axes in the case of a single crystal is theoretically possible. Thus an ideally isotropic sample (of random orientation of crystalline grains with regard to the rolling direction and area) will not bring about any change in the perpendicular component of magnetization, which is not the case with crystalline grains oriented in a given way [9].

The variation of magnetic field for Fe-Si NO strips at $f=5$, 50 and 100 Hzis representedin Figures 7 and 8. From these figures it results that, in the case of low and high magnetic flux density, the hard magnetization axis is placed at $90^{\circ}$ and the easy axis is placed at $0^{\circ}$.

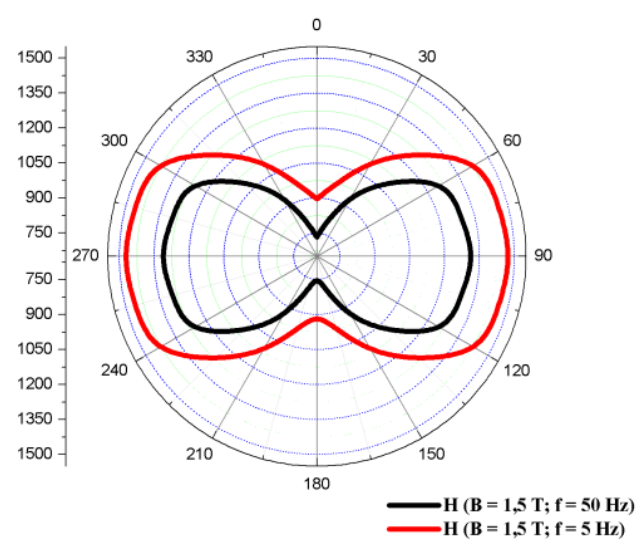

Figure 7. Comparison bet ween polar diagrams of the magnetic field in the case of Fe-Si NO strips at $B=1.5 \mathrm{~T}$ and frequencies $f=5,50 \mathrm{~Hz}$

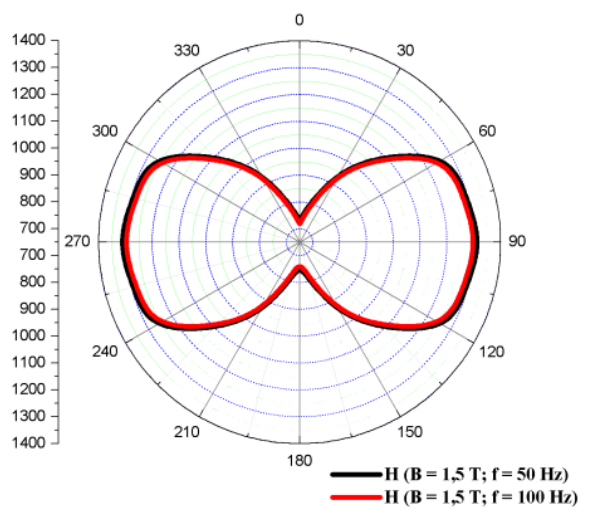

Figure 8. Comparison bet ween polar diagrams of the magnetic field in the case of Fe-Si NO strips at $B=1.5 \mathrm{~T}$ and frequencies $f=50,100 \mathrm{~Hz}$

Asimilarbehaviour of the easy and hard axeswas observedin the case of NO strips: the hard axis is placed at $90^{\circ}$ and the easy axis is placed at $0^{\circ}$ in the case of high level induction. The anisotropy is not so strong that in the case of $\mathrm{Fe}-\mathrm{Si} \mathrm{GO}$, in accordance with the theory $[3,9]$.

To better explain the results presented here, and to clarify the relationship between metallurgical structure and the anisotropy effects, on the strips (grain-oriented and non-oriented) cut parallel with the rolling direction, a doma in structure observation was performed. The domain observation was made by Kerr microscopy. The magneto-optical techniques have the advantage of being able to follow the rapid domain wall motion, even if the sample preparation is difficult and a very intense light source is needed. 
The magnetic domain structures of two Fe-Si GO strips cut at $0^{\circ}$ with the rolling direction (RD) in demagnetized statecan be observedin Figure 9. The size of the vertical side of the pictures is $2.35 \mathrm{~mm}$.
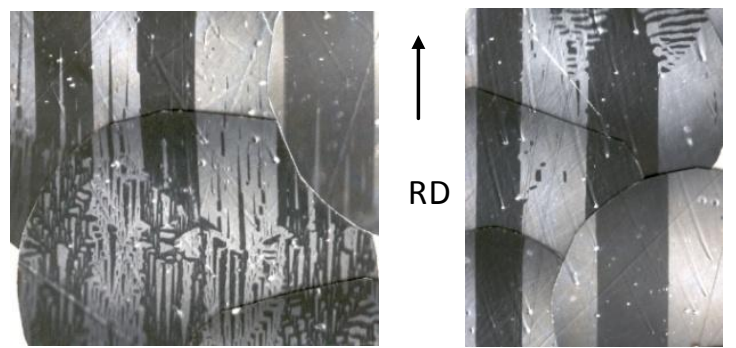

Figure 9. Magnetic domain structures in a Fe-Si GO strips in demagnetized state

In $\mathrm{Fe}-\mathrm{Si}$ grain oriented strips the first magnetocrystalline anisotropy constant $K_{1}$ is large and positive $\left(3.5 \times 10^{4}\right.$ $\left.\mathrm{J} / \mathrm{m}^{3}\right)[11]$, and therefore the $\langle 100\rangle$ directions are easy directions, since the magnetocrystalline an isotropy energy is then equal to zero.

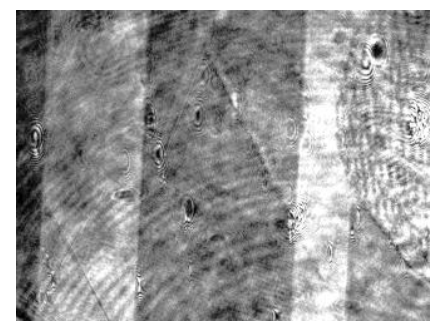

a) demagnetized state

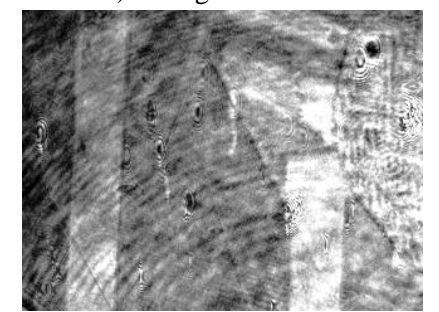

b) external magnetic field $H=87.12 \mathrm{~A} / \mathrm{m}$

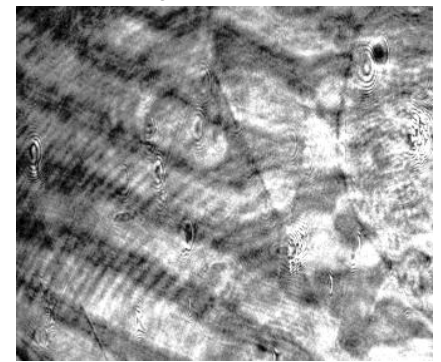

c) external magnetic field $H=293 \mathrm{~A} / \mathrm{m}$

Figure 10. Dynamic magnetic domain observation in a Fe-Si GO strip cut at $60^{\circ}$ with the rolling direction. In demagnetized state can be observed a reduce magnetic domain area, which tends to increase when an external magnetic field it is applied

The magnetocrystalline anisotropy energy is that energy which is a minimum for domains located parallel to certain crystallographic directions. The large value of $K_{1}$ forces all the domains to be parallel to $\langle 100\rangle$ directions throughout magnetization values up to the knee of the normal curve. This constraint greatly simplifies the analysis and prediction of do main structure. Figure 1 and Figure 2 show that the easy axis is parallel to the rolling direction and this observation is also sustained by domain structure observation.

In Figure 11 can be observed the magnetic domain structures of two $\mathrm{Fe}-\mathrm{Si} \mathrm{NO}$ strips cut at $0^{\circ}$ with the rolling direction (RD) in demagnetized state.
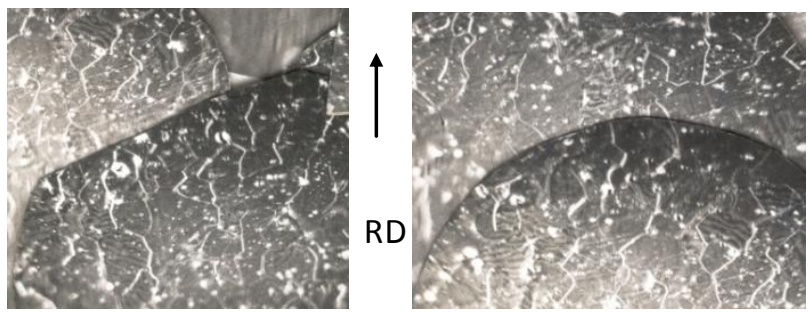

Figure 11. Magnet ic domain structures in a Fe-Si NO strips cut at $0^{\circ}$ with the rolling direction in demagnetized state

\section{Anisotropy Influence on Specific Energy Losses}

The total energy losses required for a magnetization cycle can be decomposed into the sum of hysteresis $\left(W_{h}\right)$, classical $\left(W_{c l}\right)$ and excess loss $\left(W_{e x c}\right)$ components. This separation allowstreating individually loss mechanisms that occur on different space-time scales once at a time, as if they were independent of each other.

The hysteresis loss is generated by fine-scale instabilities and can be analysed viathe coercivity mechanis ms.

In the case of eddy loss (classical loss) generated by eddy currents, the sample geometry is important. The material is treated like a homogenous medium.

The direct consequence of the magnetic domain structure is the excess losses which are very difficult to determine, as result of the great variety of do main structures.

The total energy losses in case of GO and NO strips are presented in Figures 12 and 13. One can notice that the anisotropy axis are direct related to the specific energy losses, with the lowest values at the rolling direction (GO and NO strips) and the highest values at $60^{\circ}$ in case of GO strips, respectively at $90^{\circ}$ in case of NO strips.

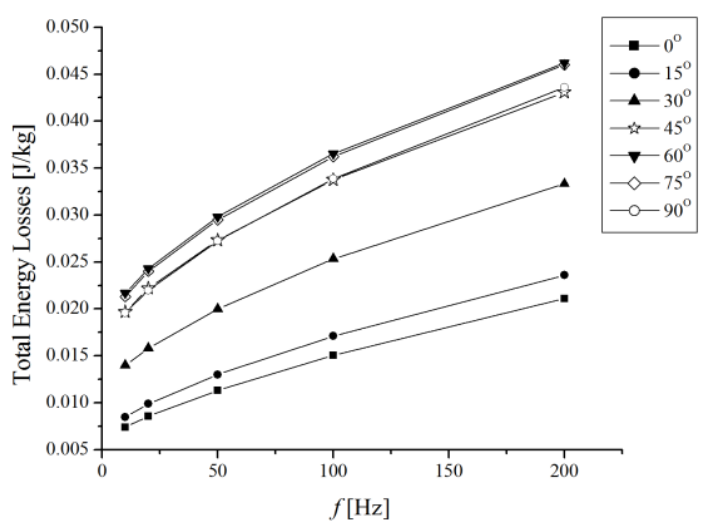

Figure 12. Specific energy losses in case of Fe-Si GO strips cut at different angles with the rolling direction at $J_{p}=1 \mathrm{~T}$ 


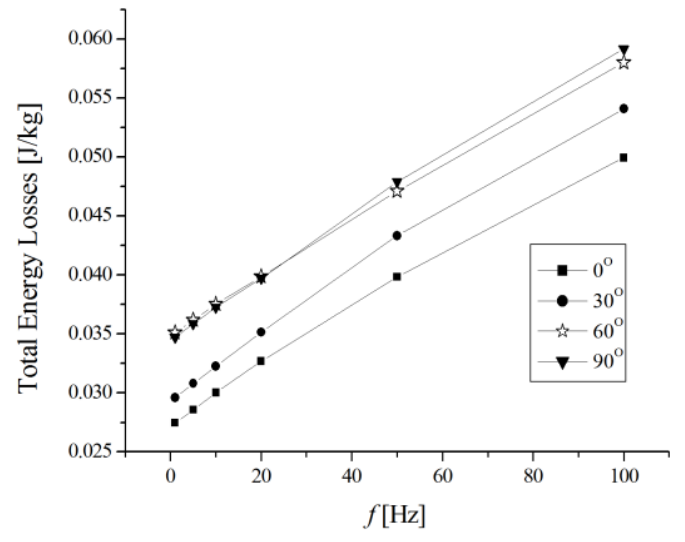

Figure 13. Specific energy losses in case of Fe-Si NO strips cut at different angles with the rolling direction at $J_{p}=1 \mathrm{~T}$

The energy loss separation was made using for the calculation of classical energy loss $\left(W_{c l}\right)$, in the approximation of linear magnetization and low frequency limit $[8,12]$, the following relation:

$$
W_{c l}=\frac{\pi^{2}}{6} \frac{\sigma J_{p}^{2} d^{2}}{\rho} f
$$

where $\sigma$ is the electric conductivity, $d$ is the thickness of the strip, $\rho$ is the density and $f$ is the frequency. Further, the excess losses $W_{\text {exc }}($ Figure 14 and 15$)$ and hysteresis losses $W_{h}$ (Figure 16 and 17) are determined as in [13, 14].

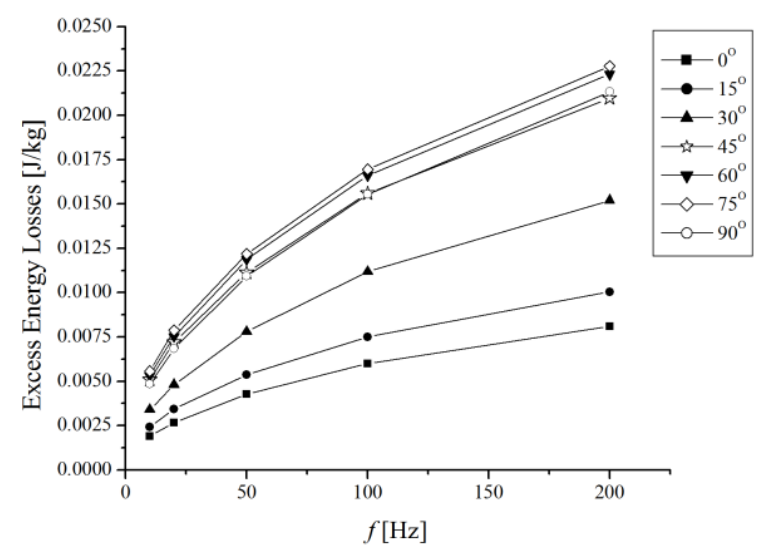

Figure 14. Variation of the excess energy losses versus frequency in the case of Fe-Si GO strips at $J_{p}=1 \mathrm{~T}$

In the case of the excess losses the influence of the orientation of the anisotropy axes is preserved (Figure 14 and 15) for both types of material. The differences between the losses at different angles are more pronounced in the case of the GO strips, as in the case of NO sheets, because of the Goss texture. Also, one can notice, in Figure 16, the same behaviour regarding the evolution of the hysteresis losses, with the lowest values for the strips cut parallel to the rolling direction, and highest values for $60^{\circ}$ (Fe-Si GO) and $90^{\circ}$ (Fe-Si NO).

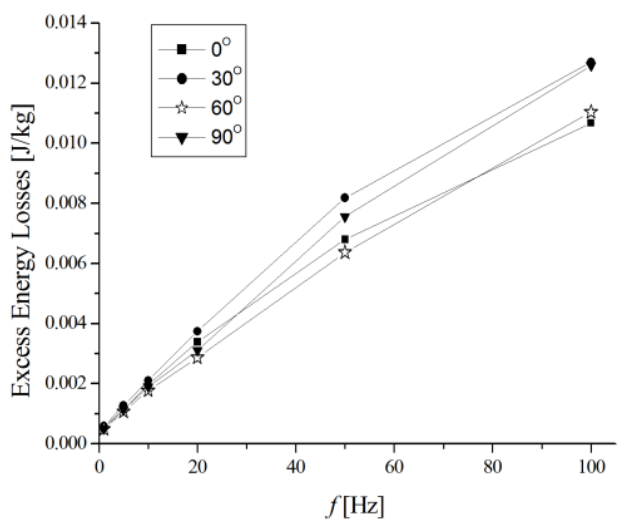

Figure 15. Variation of the excess energy losses versus frequency in the case of Fe-Si NO strips at $J_{p}=1 \mathrm{~T}$

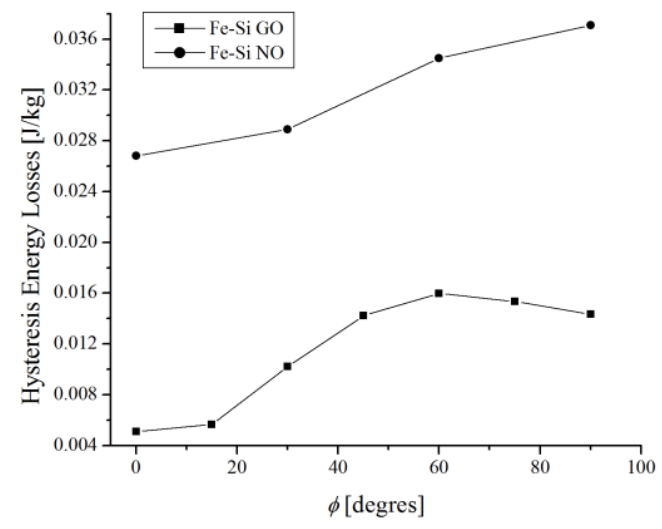

Figure 16. Variation of the hysteresis energy losses versus angle in the case of Fe-Si GO and NO strips at $J_{p}=1 \mathrm{~T}$

\section{Conclusions}

The experiments, presented in the paper, reflect the macroscopic anisotropy of the material, which is deliberately strong for the Fe-Si GO strips and must not be neglected in the case of Fe-Si NO strips. It was observed, in the case of $\mathrm{Fe}-\mathrm{Si}$ GO strips that, in the domain of low induction, the hard axis is placed at $90^{\circ}$ and not at $60^{\circ}$, because not all the grains are oriented in the lamination direction and in the range of high induction the hard axis is placed at $60^{\circ}$ and the easy axis at $0^{\circ}$. In the case of s mall magnetic fields the probable reason for the change of the hard axis from $60^{\circ}$ to $90^{\circ}$ can be associated with the magnetic domain structure of the material[10].

The rolling and the transverse directions are the only ones, for which properties can be definedindependent of the specific sampleshape[15, 16]. For a generic direction the measuring conditions (i.e.sample geometry) must be specified. Single strips are expected to display intermediate behaviours.At the magnetic flux density $B=1.5 \mathrm{~T}$ in the case of Fe-Si GO strips is obtained a polar diagram,independent 
of applied frequency (Figure 3 and 6). In the case of Fe-Si NO stripsthe polar diagrams are different atlow frequencies (Figure 7) and identically at high frequencies (Figure 8).

\section{ACKNOWLEDGEMENTS}

This work was supported by a grant of the Romanian National Authority for Scientific Research,CNDI- UEFISC DI, project nu mber PN-II-PT-PCCA- -2011-3.2-0373.

\section{REFERENCES}

[1] V. Păltânea, G. Păltânea, "Utilization of the D8 Advance diffractometer for the determination of the crystalline structure of the grain oriented and non-oriented silicon iron sheets", in Proceedings of the fifth International Workshop MMDE, pp. 102-105, Bucharest, June 2006.

[2] C. Zhiguang, N. Takahashi, B. Forghani, G. Gilbert, J. Zhang, L. Liu, Y. Fan, X. Zhang, Y. Du, J. Wang, C. Jiao, “Analy sis and Measurements of Iron Loss and Flux Inside Silicon Steel Laminations", IEEE Trans.Magn., vol. 45, no. 3, pp. 1222-1225, 2009.

[3] F. Fiorillo, C. Appino, C. Beatrice, "Magnetization process under generically directed field in $\mathrm{GOFe}-(3 \mathrm{wt} \%) \mathrm{Si}$ laminations”, J. Magn. Magn. Mater., pp. 257-260, 2002.

[4] G. Youguang, G. Z. Jian, Z. Jinjiang, "Measurement and Modeling of Rotational Core Losses of Soft Magnetic Materials Used in Electrical Machines: A Review", IEEE Trans.Magn., vol. 44, no. 2, pp. 279-291, 2008.

[5] Marian Soinski, "The Anisotropy of Coercive Force in Cold-Rolled Goss-Texture ElectricalSheets", IEEE Trans.Magn., Vol. 23, No. 6, 1987.

[6] N. Nencib, S. Spornic, A. Kedous - Lebouc, B. Cornut, "Macroscopic AnisotropyCharacterization of SiFe Using a
Rotational Single Sheet Tester", IEEE Trans.Magn., Vol. 31, No. 6, pp. 4047- 4049, November, 1995.

[7] HoriaGavrilă, WilhemKappel, MirelaCodescu, Materialema gnetice, EdituraPrintech, Romania, 2005.

[8] FaustoFiorillo, Measurement and Characterization of Magnetic Materials, Elsevier AcademicPress, USA, 2004.

[9] V. Păltânea, G. Păltânea, "Magnetic characterization of the anisotropy of the GO and NO siliconiron sheets", in Proceedings of the fifth International Workshop MMDE, pp. 106-109, ISBN: 973-718-503-X, Bucharest, June 2006.

[10] Marian Soinski, "Application of the Anisometric Method for Determining Polar Curves ofInduction, Apparent Core Loss, and Core Loss in Cold-Rolled Electrical Sheets of GossTexture", IEEE Trans.Magn., Vol. 20, No. 1, 1984.

[11] B. Fryskowski, "Experimental evaluation of magneticanisotr opy in electrical steel sheets", J. Magn. Magn. Mater., Vol. 320, no. 3, pp. 515-522, 2008.

[12] GiorgioBertotti, Hysteresis in magnetism, Academic Press, USA, 1998.

[13] G. Bertotti, "Physical interpretation of eddy current losses in ferromagnetic materials", IEEE Trans. Magn., vol. 24, no. 1, pp. 621-630, January 1988.

[14] G. Păltânea, V. Păltânea, I.V. Nemoianu, "Magnetic properties of non-oriented silicon iron sheets in case of external applied thermal treatments", Rev. Roum. Sci. Techn. Ser. Electrotechnique et Energ., tome. 55, no.4, pp.357-364, 2010.

[15] G. Korzunin, R. Puzhevich, M. Tsyrlin, "Effect of mechanical stresses on the magnetic properties of anisotropic electrical steel”, Physics of Metals and Metallography, Vol. 103, No. 2, pp. 142-151, 2007.

[16] O. Hubert, L. Daniel, "Multiscale modeling of the magneto-mechanical behavior of grain-oriented silicon steels", J.Magn.Magn.Mater., Vol. 320, no. 7, pp. 1412-1422, 2008. 